\title{
Acute Hepatic Failure and Epididymitis in a Hispanic Patient With Active Systemic Lupus Erythematosus
}

\author{
Beau M. Bailey ${ }^{\mathrm{a}}$, Kenneth S. Ramos ${ }^{\mathrm{a}, \mathrm{b}, \mathrm{c}}$, Alice Johnson ${ }^{\mathrm{a}}$, Charlene Mitchell ${ }^{\mathrm{a}}$
}

\begin{abstract}
Systemic lupus erythematosus (SLE) is an autoimmune disease known to affect a variety of organ systems. Patients with SLE are more prone to developing common infections that can mimic the complications of SLE. As such, it is essential to differentiate complications of SLE from infection to ensure appropriate management and to improve morbidity and mortality of this patient population. Here we present a 24-year-old, Hispanic male, with SLE complicated by dialysis-dependent end-stage renal disease and dilated cardiomyopathy. The patient presented to the emergency room with nausea, vomiting, and abdominal pain and admitted to the medicine service. Initial evaluation showed hypoalbuminemia coupled with elevated transaminases, INR, and total bilirubin consistent with acute liver failure. Further evaluation was negative for viral, toxic, metabolic, or vascular causes of acute failure. The patient was diagnosed with lupus hepatitis and associated acute hepatic failure, and started on high dose prednisone (60 mg daily). Complete resolution of liver function and symptoms was observed within 1 week at follow-up. The patient was readmitted 2 weeks after discharge with left scrotal pain and swelling after abruptly decreasing the prescribed prednisone dose 3 days after discharge. Physical exam and scrotal ultrasound in the emergency department were consistent with epididymitis. Urinalysis, urine culture, and gonorrhea and chlamydia PCR were all negative. Without evidence of infection, and upon reconfirmation of low serum complement levels, the patient was diagnosed with lupus epididymitis and restarted on high dose prednisone. Complete resolution of symptoms was attained within 1 week at follow-up. This case emphasizes the importance of differentiating the clinical manifestations of SLE from infection and the complexity of disease presentation in Hispanic patients.
\end{abstract}

Keywords: Systemic lupus erythematosus; Epididymitis; Hepatitis; Acute hepatic failure; Corticosteroids; Infection

Manuscript submitted May 29, 2018, accepted June 18, 2018

a Department of Medicine, University of Louisville School of Medicine, Louisville, KY 40202, USA

bDepartment of Medicine, University of Arizona, Tucson, AZ 85721-0202, USA

${ }^{\mathrm{c}}$ Corresponding Author: Kenneth S. Ramos, Department of Medicine, University of Arizona, Drachman Hall, Room B-207F, 1295 North Martin Avenue, PO Box 210202, Tucson, AZ 85721-0202, USA.

Email:ksramos@email.arizona.edu

doi: https://doi.org/10.14740/jocmr3495w

\section{Introduction}

Systemic lupus erythematosus (SLE) affects a variety of organ systems including the skin, joints, kidneys, lungs, serous membranes, and others [1]. Long-term morbidity and mortality is significantly impacted by implementation of appropriate therapy and disease management strategies. SLE patients may be more prone to the development of pneumonia and urinary tract, skin and soft tissue infections due to the underlying genetic or immunologic deficits associated with chronic immunosuppression [2]. Thus, it is essential to differentiate manifestations of SLE from concurrent infection, as the former requires immunosuppression, while the later requires appropriate antimicrobial therapy. Delays in management of either complications or infection may result in increased morbidity, long-term complications, and possible death.

\section{Case Report}

The patient is a 24-year-old, Hispanic male, with a 1-year history of SLE initially diagnosed on renal biopsy during evaluation for acute renal failure. The patient subsequently developed end-stage renal disease requiring hemodialysis. At initial presentation, he was also found to have severe dilated cardiomyopathy secondary to SLE with an ejection fraction of $17 \%$. Since initial diagnosis, treatment adherence had been poor, and the patient required multiple and repeated hospital admissions for symptoms related to end-stage renal disease and heart failure.

On the present admission, the patient presented to the emergency department with 3 days of nausea, vomiting, and abdominal pain. He also complained of increasing shortness of breath and bilateral lower extremity edema with leg pain. Emergent hemodialysis was performed to correct hyperkalemia, uremia, and hypervolemia secondary to kidney disease. The abdominal complaints were initially considered to be related to uremia, but these symptoms did not improve post dialysis. There was significant right upper quadrant tenderness on palpation without rebound tenderness, or guarding, and scleral icterus on physical examination. Laboratory examination revealed newly elevated transaminases with AST 2,365 $\mu / \mathrm{L}$ and ALT $1,315 \mu / \mathrm{L}$, which peaked at 3,594 $\mu / \mathrm{L}$ and 1,705 $\mu / \mathrm{L}$, respectively, on hospital day 1 , elevated total bilirubin at $1.5 \mathrm{mg} / \mathrm{dL}$, hypoalbuminemia of $2.2 \mathrm{~g} / \mathrm{L}$, and INR 1.9 , without anticoagulation, consistent with acute liver failure. 
Initial evaluation for acute hepatic failure revealed evidence of immunity against hepatitis A and B. The patient was false positive for hepatitis C serology secondary to his SLE, but had negative RNA titers. Acetaminophen overdose was considered, but the patient repeatedly denied use of any medications containing acetaminophen or related agents, and his toxicology screen was negative. Right upper quadrant ultrasound with normal Doppler flow excluded venous thromboembolism, as well as cirrhosis, steatosis, or a mass. Given a negative history of hypotension, ischemic hepatitis was not high in the differential, nor a metabolic etiology based on history or objective findings. A workup for autoimmune hepatitis was negative including normal anti-mitochondrial antibody and normal anti-smooth muscle antibody. The only significant objective findings were low C3 $23.6 \mathrm{mg} / \mathrm{dL}(80-150 \mathrm{mg} / \mathrm{dL})$ and C4 $3.8 \mathrm{mg} / \mathrm{dL}(15$ - $40 \mathrm{mg} / \mathrm{dL}$ ), consistent with active SLE. Of note, the anti-ribosomal-P protein was in the normal reference range.

Given these findings, the patient was diagnosed with acute liver failure secondary to lupus hepatitis and started on prednisone $60 \mathrm{mg}$ daily. Liver function improved significantly after initiation of treatment as evidenced by decreased INR to 1.3, improved transaminases with AST at $111 \mu / \mathrm{L}$ and ALT 670 $\mu / \mathrm{L}$, and compete resolution of hyperbilirubinemia, as well as symptoms at the time of discharge. At 10-day follow-up, hepatic function had returned to normal with AST at $29 \mu / \mathrm{L}$ and ALT $32 \mu / \mathrm{L}$, INR 1.1, and total bilirubin $0.6 \mathrm{mg} / \mathrm{dL}$.

The patient returned to our service with similar symptoms of shortness of breath and lower extremity edema 10 days later. On this visit, he denied abdominal pain or nausea, vomiting, or diarrhea, but had a new complaint of severe left-sided testicular pain, with scrotal edema. During the interview, it was found that the patient had not followed the steroid taper as instructed, and instead had abruptly decreased the dose to $10 \mathrm{mg}$ daily because of facial swelling. Testicular ultrasonography in the emergency department revealed bilateral epididymitis with greater involvement on the left side compared to the right. The patient denied any sexual contact over the previous year since the onset of symptoms of SLE. Urinalysis on admission was normal and the urine culture remained negative. Gonorrhea and chlamydia PCR were negative. Once again, the patient was found to have low serum complement levels with $\mathrm{C} 3$ and $\mathrm{C} 4$ at $51.3 \mathrm{mg} / \mathrm{dL}$ and $8.8 \mathrm{mg} / \mathrm{dL}$, respectively. Based on these findings, the patient was diagnosed with lupus epididymitis given a recent history of active disease with multiple organ system involvement, likely precipitated by the abrupt reduction in his steroid dose. We restarted the patient on high dose steroids with plans for outpatient follow-up and transition to a steroid sparing regimen that would facilitate patient compliance. On 1-week follow-up post discharge, testicular pain and edema had completely resolved and the patient had been compliant with treatment.

\section{Discussion}

Hepatitis is a relatively rare complication of SLE, occurring in approximately $4.7 \%$ of lupus patients [3]. Most frequently lupus hepatitis presents as a chronic, often asymptomatic hepa- titis. Hepatitis can also result from steatosis secondary to corticosteroid treatment. Acute liver failure appears to be a rare complication of lupus hepatitis [4], but several case reports have appeared in the literature of severe liver involvement requiring transplantation $[5,6]$. Clinical manifestations of lupus hepatitis and acute liver failure respond well to high dose prednisone treatment, most frequently without progression to end-stage liver disease [4]. Our patient presented with symptoms and objective findings consistent with acute liver failure, and the workup was negative for viral, toxic, metabolic, and vascular etiologies. Markedly decreased complement C3 and C4 were consistent with active SLE. Although the anti-ribosomal-P protein in our patient was normal, he responded well to high dose corticosteroid treatment, with complete resolution of symptoms and objective findings within weeks of treatment. There is some controversy about the utility of anti-ribosomal$\mathrm{P}$ protein in assessing lupus hepatitis with some evidence of strong correlation [7], and other studies reporting limited utility in diagnosing lupus hepatitis [8]. The effective response to prednisone treatment is consistent with an immunologic etiology, as opposed to etiologies associated with infectious agents or adverse drug reaction.

Epididymitis is a known rare complication of other systemic vascular diseases including Henoch-Schonlein purpura $[9,10]$, Wegener granulomatosis [11], polyarteritis nodosa [12], and more frequently Behcet disease [13, 14]. Epididymitis is a rarely seen complication of active SLE, with only three prior case reports identified in the literature [15-17]. In two of these cases, testicular pain from epididymis involvement was one of the primary symptoms of the disease at the time of presentation. In one of these cases, diagnosis of SLE was made on renal biopsy as part of the workup for acute renal failure. In the other case, the testicle was resected due to concern for tumor and the pathologic diagnosis was lupus epididymitis. Our patient presented with symptoms and objective findings consistent with lupus epididymitis shortly after abruptly stopping therapeutic prednisone treatment for active lupus hepatitis. Infectious etiologies were ruled out prior to restarting high dose steroid treatment. At 1-week follow-up after discharge, the patient had been compliant with treatment and his symptoms had completely resolved.

A review of worldwide epidemiology has indicated that susceptibility to SLE has a strong genetic component with the highest susceptibility seen in non-European groups. Black, Asians and Hispanics with SLE tend to develop more severe disease and a greater number of manifestations and organ involvement, even after accounting for socio-economic factors [18]. Thus, the increased genetic risk burden associated with increased autoantibody reactivity in Hispanic patients may explain the severity of the phenotype observed in our case. Because Hispanics are an admixed population, it is possible that the presence of high susceptibility genes can be used in the future to identify those at highest risk. A study by Kheir et al [19] presented evidence that SLE patients of Native American ancestry meet the SLE classification at a younger age than European American counterparts and were more likely to have higher antinuclear antibody titers and autoantibodies of unknown specificities. In another study, the impact of admixture in a cohort of 1,125 Hispanics was examined where the best- 
fitted model was a four-population model with average ancestry of European (48\%), American Indian (40\%), African (8\%) and a fourth unknown population at (4\%). Of note were the findings that a significantly enhanced risk was associated with American Indian ancestry and that Integrin alpha M (ITGAM), one of the protein subunits that forms the complement receptor 3 (CR3), is associated with SLE [20]. Future studies focusing on gene-lifestyle-environment interactions that influence SLE clinical outcomes are required.

\section{Conclusions}

Hepatitis is a relatively rare complication of SLE that usually presents as chronic hepatitis or steatosis secondary to corticosteroid treatment. The patient in our case presented with symptoms and objective finding consistent with acute liver failure with complete clinical resolution after treatment with high dose oral corticosteroids. Epididymitis is also an extremely rare complication of SLE with only a few single case reports documented. The occurrence of epididymitis after abrupt cessation of high dose oral corticosteroid treatment for active lupus hepatitis may be of relevance to the future management of young males with SLE. After ruling out infectious causes, the patient responded well to the reintroduction of high dose oral prednisone, with complete resolution of symptoms within 1 week. This case emphasizes the importance of differentiating the clinical manifestations of SLE from infection and raises important questions about the extent to which genetic admixture contributes to the course of disease.

\section{References}

1. Cervera R, Khamashta MA, Font J, Sebastiani GD, Gil A, Lavilla P, Mejia JC, et al. Morbidity and mortality in systemic lupus erythematosus during a 10-year period: a comparison of early and late manifestations in a cohort of 1,000 patients. Medicine (Baltimore). 2003;82(5):299308.

2. Zandman-Goddard G, Shoenfeld Y. Infections and SLE. Autoimmunity. 2005;38(7):473-485.

3. Ebert EC, Hagspiel KD. Gastrointestinal and hepatic manifestations of systemic lupus erythematosus. J Clin Gastroenterol. 2011;45(5):436-441.

4. Piga M, Vacca A, Porru G, Cauli A, Mathieu A. Liver involvement in systemic lupus erythematosus: incidence, clinical course and outcome of lupus hepatitis. Clin Exp Rheumatol. 2010;28(4):504-510.

5. Atsumi T, Sagawa A, Jodo S, Amasaki Y, Nakabayashi T, Ohnishi K, Fujisaku A, et al. Severe hepatic involvement without inflammatory changes in systemic lupus erythematosus: report of two cases and review of the literature. Lupus. 1995;4(3):225-228.

6. Zazzetti F, Buschiazzo A, Villamil FG, Barreira JC. Liver transplantation in systemic lupus erythematosus: case report and review of the literature. Lupus. 2011;20(1):9094.

7. Arnett FC, Reichlin M. Lupus hepatitis: an under-recognized disease feature associated with autoantibodies to ribosomal P. Am J Med. 1995;99(5):465-472.

8. Khalifa M, Benjazia E, Rezgui A, Ghannouchi N, Alaoua A, Braham A, Letaief A, et al. [Lupus hepatitis: a case series of 12 patients]. Rev Med Interne. 2011;32(6):347349.

9. Lee JS, Choi SK. Acute scrotum in 7 cases of SchoenleinHenoch syndrome. Yonsei Med J. 1998;39(1):73-78.

10. Sakanoue M, Higashi Y, Kawai K, Sugita S, Kanekura T. Henoch-Schonlein purpura with epididymitis in an adult. J Dermatol. 2011;38(6):620-622.

11. Miller DC, Koss MN. Wegener granulomatosis presenting as epididymitis. Urology. 2009;73(6):1225-1226.

12. Coulomb MA, Bagneres D, Rossi D, Bastide C. [Epididymitis: Unusual presentation of periarteritis nodosa]. Prog Urol. 2010;20(4):311-313.

13. Cho YH, Jung J, Lee KH, Bang D, Lee ES, Lee S. Clinical features of patients with Behcet's disease and epididymitis. J Urol. 2003;170(4 Pt 1):1231-1233.

14. Yilmaz O, Yilmaz S, Kisacik B, Aydogdu M, Bozkurt Y, Erdem H, Pay S, et al. Varicocele and epididymitis in Behcet disease. J Ultrasound Med. 2011;30(7):909-913.

15. Kuehn MW, Oellinger R, Kustin G, Merkel KH. Primary testicular manifestation of systemic lupus erythematosus. Eur Urol. 1989;16(1):72-73.

16. MacIver H, Hordon L. An unusual case of testicular pain. Clin Rheumatol. 2009;28(3):351-352.

17. Walker G, Merry P, Sethia K, Ball RY. A case of testicular lupus. Lupus. 2000;9(5):397-398.

18. Lewis MJ, Jawad AS. The effect of ethnicity and genetic ancestry on the epidemiology, clinical features and outcome of systemic lupus erythematosus. Rheumatology (Oxford). 2017;56(suppl_1):i67-i77.

19. Kheir JM, Guthridge CJ, Johnston JR, Adams LJ, Rasmussen A, Gross TF, Munroe ME, et al. Unique clinical characteristics, autoantibodies and medication use in Native American patients with systemic lupus erythematosus. Lupus Sci Med. 2018;5(1):e000247.

20. Molineros JE, Kim-Howard X, Deshmukh H, Jacob CO, Harley JB, Nath SK. Admixture in Hispanic Americans: its impact on ITGAM association and implications for admixture mapping in SLE. Genes Immun. 2009;10(5):539545. 